\section{BRAZIULIAN JOURNAL \\ OF MEDICAL AND BIOLOGICAL RESEARCH}

www.bjournal.com.br
ISSN 1414-431X

Volume 45 (12) 1102-1340 December 2012

\section{BIOMIDICAL SCIENCES}

AND

CLINICAL INVESTIGATION

Braz J Med Biol Res, December 2012, Volume 45(12) 1308-1314

doi: 10.1590/S0100-879X2012007500165

Circulating levels of inflammation-associated miR-155 and endothelial-enriched miR-126 in patients with end-stage renal disease

Honglei Wang, Wujian Peng, Xuemei Shen, Yunhui Huang, Xin Ouyang and Yong Dai

The Brazilian Journal of Medical and Biological Research is partially financed by

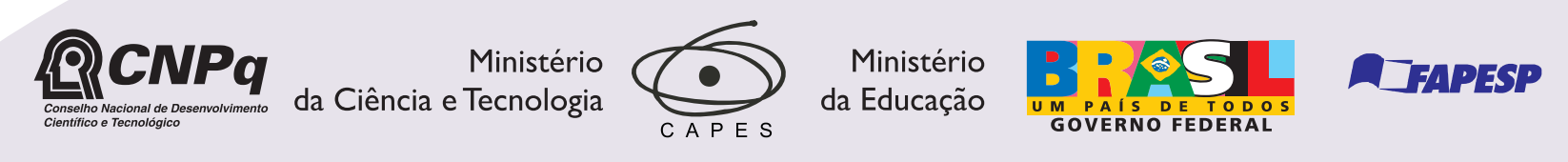

Sciefo
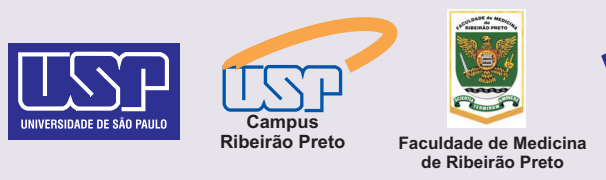

Institutional Sponsors

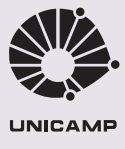

SHIMADZU

$\oplus$

UNICAMP
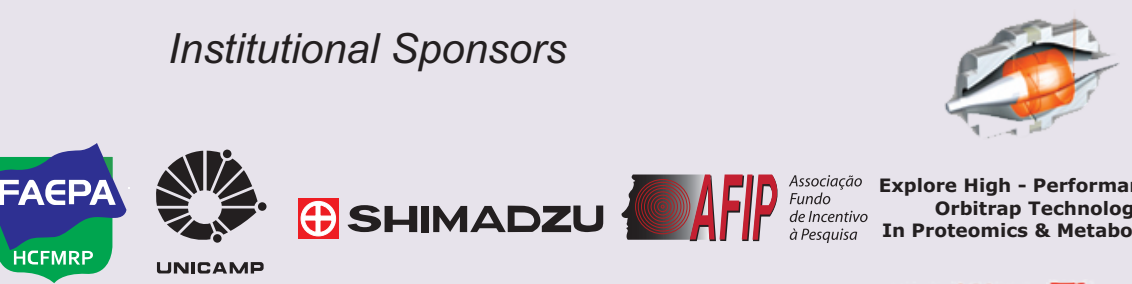

GOVERNO FEDERAL 


\title{
Circulating levels of inflammation-associated miR-155 and endothelial-enriched miR-126 in patients with end-stage renal disease
}

\author{
Honglei Wang, Wujian Peng, Xuemei Shen, Yunhui Huang, Xin Ouyang and Yong Dai \\ Clinical Medical Research Center, Second Clinical Medical College, Shenzhen People's Hospital, \\ Jinan University, Shenzhen, Guangdong, China
}

\begin{abstract}
Circulating microRNAs (miRNAs) may represent a potential noninvasive molecular biomarker for various pathological conditions. Moreover, the detection of circulating miRNAs can provide important novel disease-related information. In particular, inflammation-associated miR-155 and endothelial-enriched miR-126 are reported to be associated with vascular homeostasis. Vascular damage is a common event described in end-stage renal disease (ESRD). We hypothesized that miR-155 and miR-126 may be detectable in the circulation and serve as potential biomarkers for risk stratification. In this study, we assessed miR-155 and miR-126 in the plasma of 30 ESRD patients and 20 healthy controls using real-time quantification RT-PCR. The circulating levels of miR-155 and miR-126 were significantly reduced in patients with ESRD compared to healthy controls. However, there was no significant difference of circulating miR-155 and miR-126 levels between prehemodialysis and posthemodialysis patients. Furthermore, both circulating miR-126 and miR-155 correlated positively with estimated glomerular filtration rate (miR-126: $r$ $=0.383, \mathrm{P}=0.037 ;$ miR-155: $r=0.494, \mathrm{P}=0.006$ ) and hemoglobin (miR-126: $r=0.515, \mathrm{P}=0.004 ; \mathrm{miR}-155: r=0.598, \mathrm{P}<$ 0.001 ) and correlated inversely with phosphate level (miR-126: $r=-0.675, P<0.001$; miR-155: $r=-0.399, P=0.029$ ). Pearson's correlation was used to compare circulating levels of miRNAs with clinical parameters. These results suggested that circulating miR-155 and miR-126 might be involved in the development of ESRD. Further studies are needed to demonstrate the role of circulating miR-155 and miR-126 as candidate biomarkers for risk estimation.
\end{abstract}

Key words: MicroRNAs; End-stage renal disease; Hemodialysis; MiR-126; MiR-155

\section{Introduction}

MicroRNAs (miRNAs) are a class of small ( 22 nucleotides) non-coding RNAs that regulate gene expression at the post-transcriptional level by either translational repression or messenger RNA degradation (1). MiRNAs have been shown to play critical roles in various cellular processes, inflammation, and angiogenesis (2). In particular, miR-126 and miR-155 have been reported to control vascular homeostasis, angiogenesis, and vascular repair. In addition, miR-155, an important multifunctional miRNA, has been shown to regulate the endothelial inflammatory response and migration mediated by angiotensin $\alpha$ (3). Moreover, accumulating evidence points to an essential role of miRNAs in kidney disease, which is supported by the finding that the depletion of the miRNA-processing enzyme Dicer results in defects in renin cells (juxtaglomerular cells), angiogenesis, and renal morphological integrity and function (4-6). Specific miRNA expression profiles have been described for diabetic nephropathy, polycystic kidney disease and kidney allograft rejection (7).

Cardiovascular diseases are the leading cause of morbidity and mortality in patients with end-stage renal disease (ESRD) (8), and this cardiovascular risk cannot be completely elucidated by traditional risk factors. Thus, more emphasis has been placed on non-traditional risk factors such as vascular calcification, inflammation, endothelial dysfunction and oxidative stress, and sympathetic overactivation $(9,10)$. A few specific miRNAs have been implicated in the functional regulation of endothelial cells (ECs), as well as inflammatory responses and peripheral angiogenic signaling (11). Thus, differentially expressed miRNAs are responsible for non-traditional risk factors of chronic kidney disease (CKD). Given that miR-126 and

Correspondence: Yong Dai, Clinical Medical Research Center, Second Clinical Medical College, Shenzhen People's Hospital, Jinan University, Shenzhen, Guangdong, 518020, China. Fax: +86-0755-2562-6750. E-mail: daiyong22@yahoo.com.cn

Received February 24, 2012. Accepted August 27, 2012. Available online October 19, 2012. Published December 17, 2012. 
miR-155 play important roles in EC functional regulation, endothelial dysfunction has been reported to be associated with ESRD. Therefore, we hypothesized that miR-126 and miR-155 may be involved in vascular damage-related pathophysiology in patients with ESRD.

MiRNAs were previously considered to act as intracellular RNAs to regulate gene expression. However, increasing evidence suggested that miRNAs can be detected in the circulation in remarkably stable form that withstands repeated freezing/thawing cycles (12). It was discovered that miRNAs circulate within membrane-bound vesicles that are resistant to RNase digestion (13). One study additionally demonstrated that circulating Argonaute 2 complexes, carrying a number of miRNAs independent of vesicles, are one mechanism responsible for the stability of circulating miRNAs (14). Similarly, it has been recognized that specific miRNAs could serve as potential biomarkers of various diseases in the circulation. Therefore, in the present study, we assayed the circulating levels of inflammation-associated miR-155 and endothelial-enriched miR-126 in patients with ESRD, expecting to be able to provide new insights into the development of ESRD and the manifestation of its vascular complications.

\section{Material and Methods}

\section{Patients and volunteers}

The study included ESRD patients of $\geq 18$ years of age who had been receiving maintenance hemodialysis 3 times weekly for at least 3 months. The primary disease in ESRD patients was chronic glomerulonephritis in China, which was proved by biopsy. Twenty healthy adults without any evidence of CKD or inflammatory disorders were included in the control group. All participating subjects gave written informed consent before participating in the study. All study protocols and consent forms were approved by the Second Clinical Medical College (Shenzhen People's Hospital) of Jinan University, which abides by the Helsinki Declaration on ethical principles for medical research involving human subjects.

\section{Blood processing}

In the pre-hemodialysis (pre-HD) patients, whole blood samples $(3 \mathrm{~mL})$ were collected immediately pre-HD from fistula needles before machine connection or any heparin exposure. In the post-hemodialysis (post-HD) patients, samples were also collected from fistula needles. All blood samples were processed for the isolation of plasma within $4 \mathrm{~h}$ of collection. The blood was centrifuged at $2000 \mathrm{~g}$ for $10 \mathrm{~min}$ at room temperature. Plasma was then transferred to RNase-free tubes and stored at $-80^{\circ} \mathrm{C}$.

\section{RNA isolation}

RNA was isolated by using a Trizol-based miRNA isolation protocol in which $300 \mu \mathrm{L}$ plasma was mixed with
$1200 \mu \mathrm{L}$ Trizol (Invitrogen, USA). After the addition of Trizol, we supplemented the plasma with $2 \mu \mathrm{L}$ synthetic cel-lin-4 (Shanghai GenePharma, China) and subsequently mixed it with $200 \mu \mathrm{L}$ chloroform. The organic and aqueous phase was separated by centrifugation. The aqueous phase containing the RNA was carefully removed, and RNA was precipitated as described previously $(15,16)$. RNA was solubilized by the addition of 25 $\mu \mathrm{L}$ RNase-free water.

\section{Real-time quantification RT-PCR (RT-qPCR) of mature miRNAs}

To determine the regulation of miRNAs, we performed RT-qPCR using RNA isolated from 20 healthy controls and 30 pre-HD and post-HD patients. Targetspecific stem-loop RT primers that bind to the $3^{\prime}$ portion of miRNA molecules were used and reverse transcription was performed using M-MLV Reverse Transcriptase (Promega, USA). Real-time PCR was then performed using an ABI PRISM 7500 Sequence Detection System (Applied Biosystems, USA) with miRNA-specific forward and reverse primers. Each SYBR Green reaction was performed with $5 \mu \mathrm{L}$ template cDNA, $10 \mu \mathrm{L} 2 \mathrm{X}$ SYBR Greens PCR Master Mix (Toyobo, Japan), $0.5 \mu \mathrm{L}$ of each primer $(10 \mu \mathrm{mol} / \mu \mathrm{L})$, and water to adjust to a final volume of $20 \mu \mathrm{L}$.

The miRNA-specific forward primer sequences were designed based on the mature miRNA sequence obtained from the miRBase database (http://www.mirbase. org/). The RT primers used include 1) RT forward: hsamiR-126, 5'-ACACTCCAGCTGGGTCGTACCGTGAGT AATAA-3'; hsa-miR-155, 5'-ACACTCCAGCTGGGTT AATGCTAATCGTGATA-3'; cel-lin-4, 5'-ACACTCCAG CTGGGTCCCTGAGACCTCAAGTG-3', and 2) miRNA reverse: 5'-CTCAACTGGTGTCGTGGA-3'. All reactions were incubated on a 96 -well plate at $95^{\circ} \mathrm{C}$ for $5 \mathrm{~min}$, followed by 40 cycles of $95^{\circ} \mathrm{C}$ for $15 \mathrm{~s}, 65^{\circ} \mathrm{C}$ for $15 \mathrm{~s}, 72^{\circ} \mathrm{C}$ for $32 \mathrm{~s}$. Each sample was assayed in triplicate for the analysis. Finally, at the end of the PCR cycles, melting curves were constructed to confirm the specificity of the expected PCR products.

\section{Statistical analysis}

All data for continuous variables are reported as means $\pm \mathrm{SD}$, unless otherwise stated. Values were normalized to cel-lin-4 and were calculated according to the $2^{-\Delta \Delta C T}$ method, as described previously (17). For group-wise comparisons, the Student $t$-test (2 groups), ANOVA, or Kruskal-Wallis test (n group) were used, as appropriate. The Pearson product-moment test was used to examine the correlations between miRNA levels and clinical parameters. All statistical tests were performed 2-sided and the level of significance was set at $P<0.05$. All statistical analyses were carried out using the SPSS (version 13.0) software. 


\section{Results}

A total of 50 subjects were studied, including 30 ESRD patients and 20 healthy controls. There was no difference in age between ESRD patients and healthy controls $(P>$ $0.05)$. The clinical characteristics of the study population are summarized in Table 1.

\section{Expression of miRNAs in plasma}

To date, no reliable endogenous control miRNA has been established and validated to normalize for miRNA content. The use of synthetic versions of Caenorhabditis elegans miRNAs as controls has been proposed by many investigators (15). Therefore, we supplemented the samples with recombinant cel-lin-4, which can be consistently detected by RT-qPCR, and no significant difference in raw $\mathrm{Ct}$ values of cel-lin-4 was detected among the 3 groups ( $P=0.754$; Kruskal-Wallis test). Thus, cel-lin-4 was used to normalize the differences in the efficiency of RNA isolation.

As shown in Figure 1, the circulating levels of miR-126 and miR-155 were significantly different when ESRD patients were compared to healthy controls. The circulating levels of miR-126 and miR155 were significantly reduced in pre-HD patients compared to healthy controls. However, no significant internal correlation was observed between the circulating levels of miR-126 and miR-155 ( $r$ $=-0.179, P=0.450)$. Interestingly, the circulating levels of miR-126 and miR-155 were still significantly reduced in post-HD patients compared to healthy controls (miR-126: $\mathrm{P}<0.001$; miR-155: $\mathrm{P}<0.001$ ). Remarkably, no significant difference was observed between pre-HD and post-HD patients $(P>0.05)$.

\section{Correlations between miRNA level and clinical parameters}

The relative expression levels of circulating miRNAs were calculated by the comparative $2^{-\Delta \Delta C T}$ method. Figure 2 shows that the circulating levels of miR-126 correlated positively with both estimated glomerular filtration rate (eGFR) $(r=0.383, P=0.037)$ and hemoglobin $(r=0.515, P=0.004)$. However, they correlated negatively with phosphate level $(r=-0.675, P<0.001)$ and were not significantly correlated with C-reactive protein (CRP) $(r=-0.234, P=0.214)$. As shown in Figure 3, the circulating miR-155 levels correlated positively with both eGFR $(r=0.494, P=0.006)$ and hemoglobin $(r=0.598, P<0.001)$, but correlated negatively

Table 1. Baseline clinical data of the subjects.

\begin{tabular}{lcc}
\hline & $\begin{array}{c}\text { End-stage renal disease } \\
(\mathrm{N}=30)\end{array}$ & $\begin{array}{c}\text { Healthy controls } \\
(\mathrm{N}=20)\end{array}$ \\
\hline Age (years) & $52.17 \pm 11.73$ & $49.25 \pm 11.25$ \\
Males & $73 \%$ & $60 \%$ \\
Chronic glomerulonephritis & $100 \%$ & - \\
Blood pressure & & \\
$\quad$ Systolic (mmHg) & $144 \pm 15.62^{*}$ & $125 \pm 10$ \\
Diastolic $(\mathrm{mmHg})$ & $87 \pm 10.5$ & $84 \pm 7$ \\
eGFR (mL/min) & $8.38 \pm 1.97$ & - \\
Hemoglobin $(\mathrm{mg} / \mathrm{dL})$ & $91 \pm 13.61^{*}$ & $116 \pm 9.5$ \\
Albumin $(\mathrm{g} / \mathrm{dL})$ & $3.6 \pm 0.6^{*}$ & $4.6 \pm 0.6$ \\
CRP (mg/L) & $10.89 \pm 9.1^{*}$ & $1.12 \pm 0.48$ \\
Calcium (mM) & $2.26 \pm 0.38$ & $2.31 \pm 0.29$ \\
Phosphate $(\mathrm{mM})$ & $2.1 \pm 0.4^{*}$ & $1.2 \pm 0.19$ \\
\hline
\end{tabular}

Data are reported as means \pm SD. eGFR $=$ estimated glomerular filtration rate; $\mathrm{CRP}=\mathrm{C}$-reactive protein. ${ }^{*} \mathrm{P}<0.05$ compared to healthy controls (Student $t$-test).
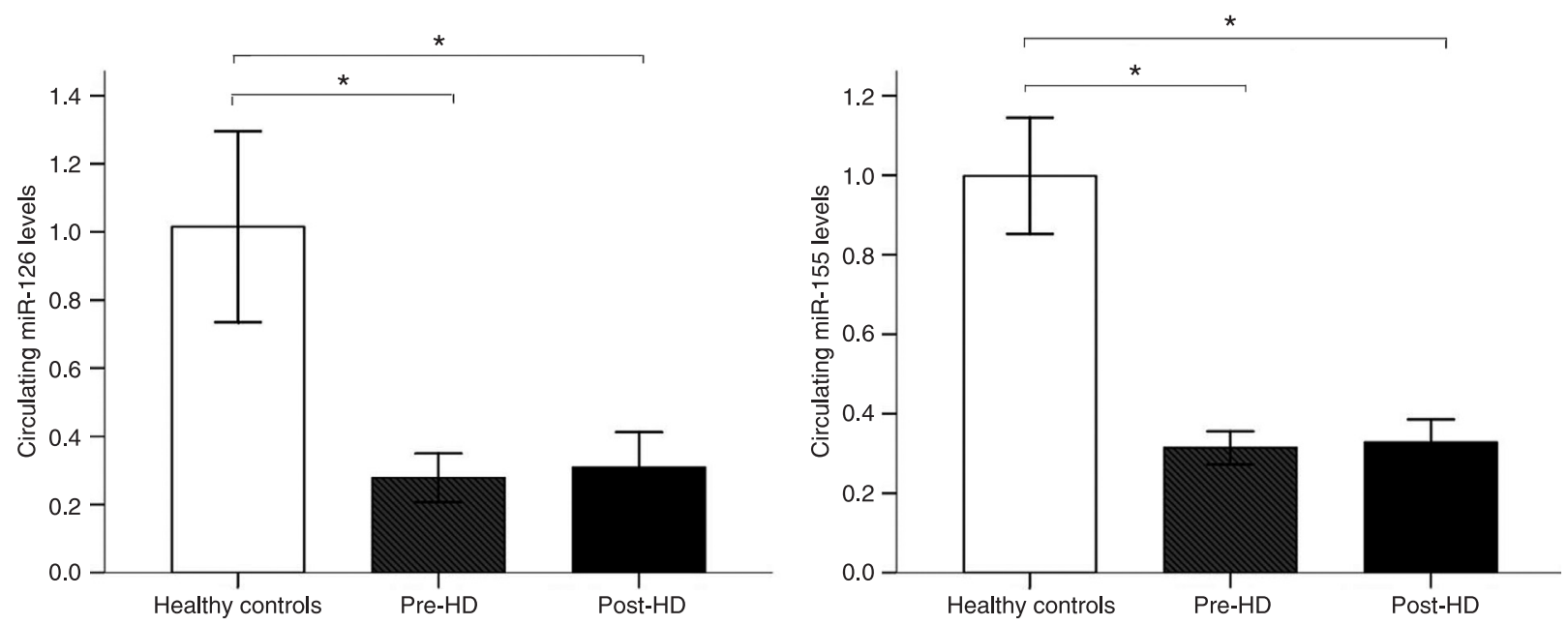

Figure 1. Circulating miRNAs in patients with end-stage renal disease (ESRD) and healthy controls. Circulating levels of miR-126 and miR-155 in plasma were obtained from patients with ESRD $(\mathrm{N}=30)$ and healthy controls $(\mathrm{N}=20)$ by RT-qPCR. Plasma miR-126 and miR-155 levels were calculated by the $2^{-\Delta \Delta C T}$ method. ${ }^{*} \mathrm{P}<0.001$ (ANOVA followed by the Tukey post-test). 

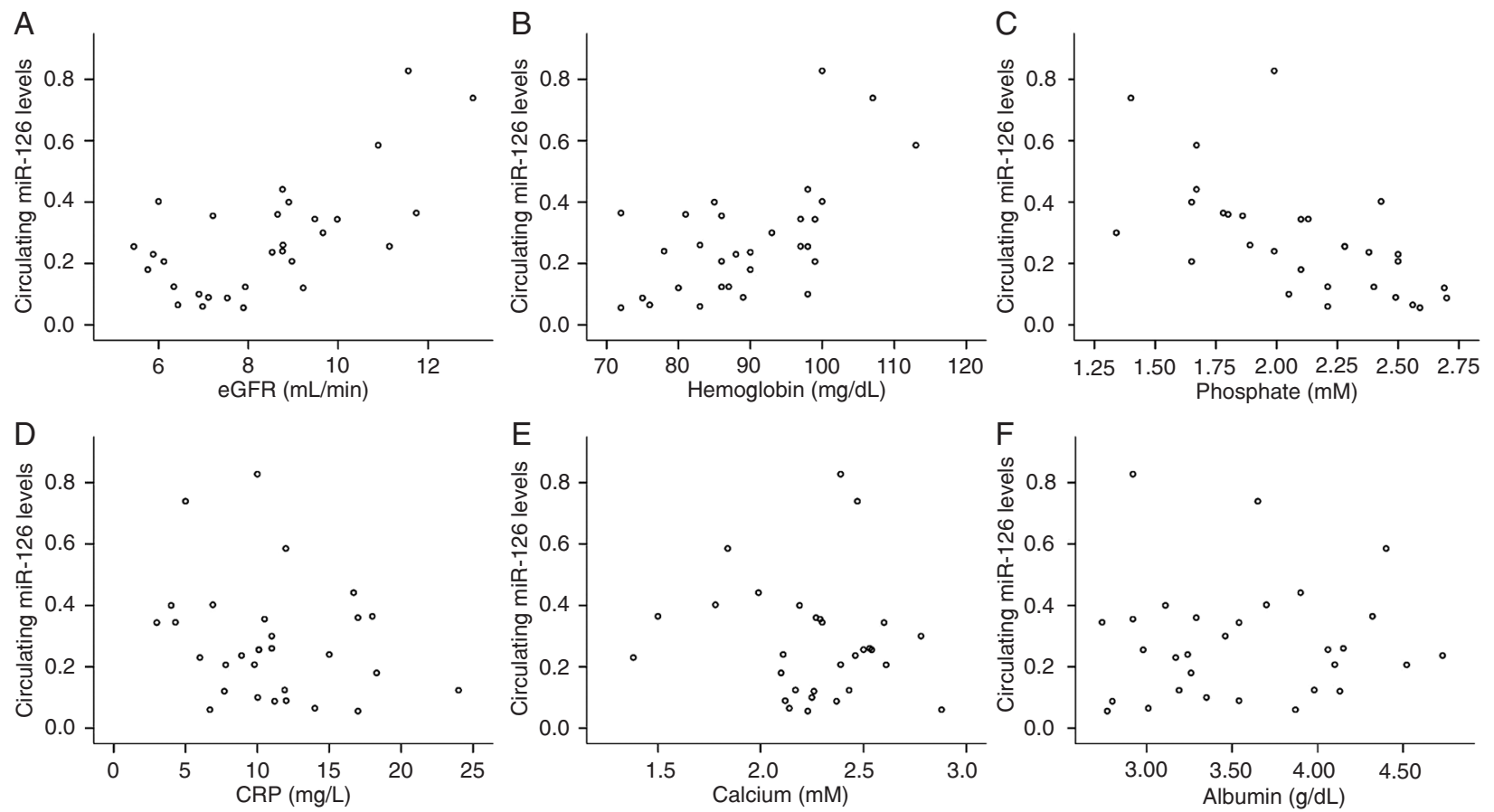

Figure 2. Correlations between circulating miR-126 and $A$, estimated glomerular filtration rate (eGFR); $B$, hemoglobin; $C$, phosphate; $D$, C-reactive protein (CRP); $E$, calcium; $F$, albumin. Statistical analysis was performed by the Pearson correlation test.
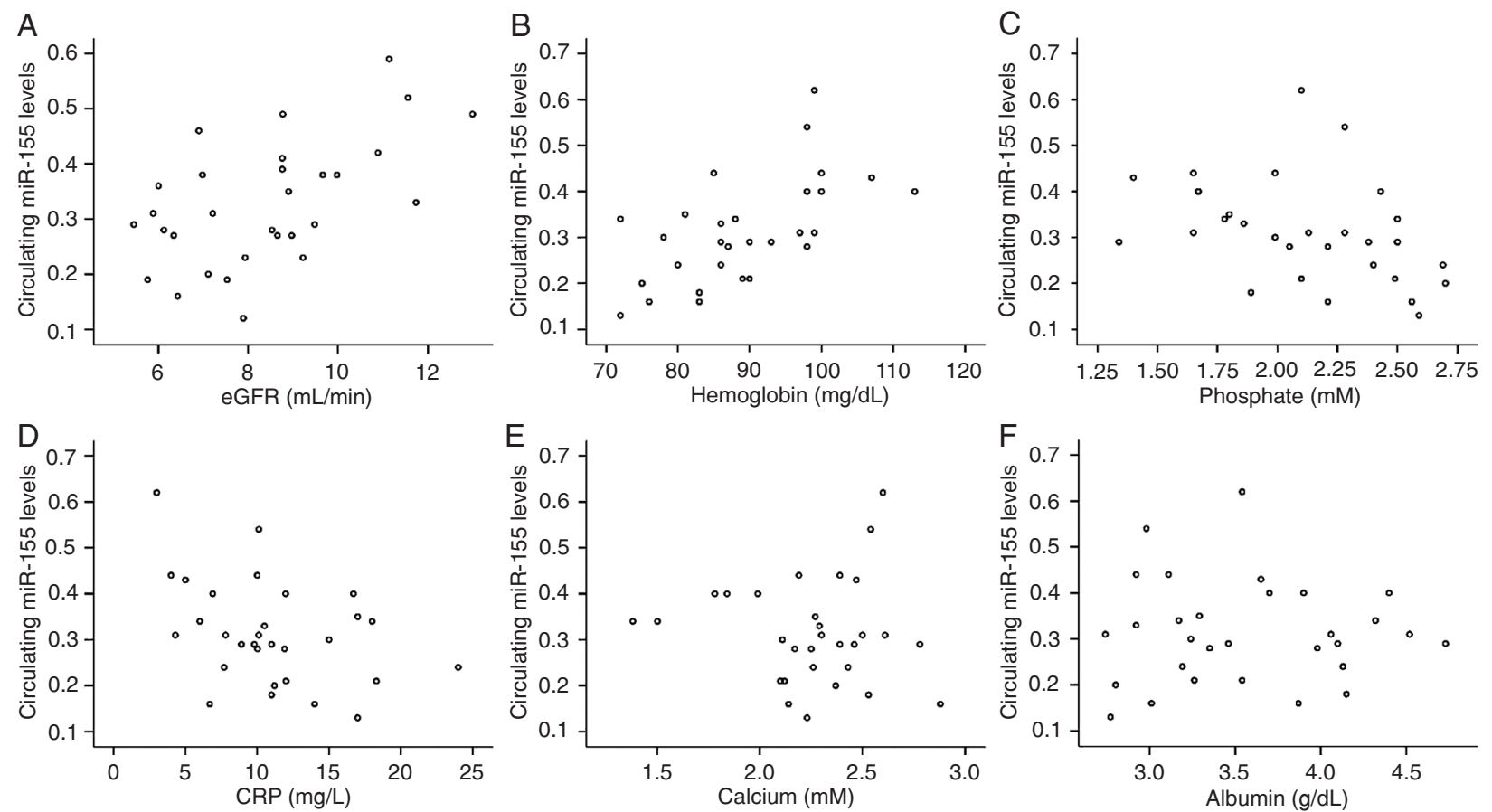

Figure 3. Correlations between circulating miR-155 and $A$, estimated glomerular filtration rate (eGFR); $B$, hemoglobin; $C$, phosphate; $D$, C-reactive protein (CRP); $E$, calcium; $F$, albumin. Statistical analysis was performed by the Pearson correlation test. 
with phosphate levels $(r=-0.399, P=0.029)$ and CRP $(r$ $=-0.379, P=0.039$ ). In addition, the circulating levels of miR-126 and miR-155 were not significantly correlated with calcium or albumin levels.

\section{Discussion}

We showed here that the circulating levels of endothelial-enriched and inflammation-associated miRNAs were significantly dysregulated in patients with ESRD receiving maintenance hemodialysis compared to healthy controls; moreover, the levels of these miRNAs tended to be significantly reduced in patients with ESRD. Our findings warrant prospective investigation into the role of circulating miRNAs in the pathophysiology and cardiovascular complications of ESRD.

\section{MiR-126 and miR-155 in ESRD}

It is important to realize that endothelial dysregulation starts in the early stage of CKD (18) and is a common event identified in both chronic and acute renal failure and in ESRD (9). In addition, studies have suggested that inflammation exists in patients with ESRD and might be aggravated by hemodialysis (19). Similarly, recent studies have demonstrated that miRNA can regulate vascular inflammation, endothelial homeostasis, and angiogenesis. In particular, miR-126 and miR-155 have been shown to be involved in vascular dysfunction and inflammation. MiR-126 is highly enriched in ECs and endothelial apoptotic bodies and governs the maintenance of vascular integrity and angiogenesis $(20,21)$. Moreover, miR-126 can facilitate vascular endothelial growth factor signaling pathway (11) and modify vascular inflammation by suppressing leukocyte adhesion to ECs. MiR-155 regulates the expression of adhesion molecules in inflammatory ECs as well as inflammation response in ECs mediated by and angiotensin $\alpha$ (22). Another study revealed that miR-155 and angiotensin $\alpha$ type 1 receptor (AT1R) are co-expressed in ECs and vascular smooth-muscle cells (23) and their expression is negatively correlated with the expression of the endogenous AT1R (24). A silent polymorphism (+1166 $A / C$ ) in the human AT1R has been shown to be associated with cardiovascular disease (CVD), which might be mediated by enhanced AT1R activity (23). Therefore, miR-126 and miR-155 might be responsible for endothelial activation and increased CVD risk in ESRD patients (25).

\section{Circulating miR-155 and miR-126 in ESRD patients}

Recent studies have revealed that miRNAs previously identified in specific cells can also be detected in the circulation. Neal et al. (26) have shown that circulating levels of total and specific miRNAs were reduced in patients with severe chronic renal failure, with important implications for the use of circulating miRNAs as biomarkers in patients with renal impairment and for the pathogenesis of uremia.
Moreover, Amabile et al. (27) suggested that miR-210 might serve as a strong and independent predictor of survival in critically ill patients with acute kidney injury. In the present study, we measured the expression of miR-155 and miR126 , which were significantly reduced in ESRD patients. Our data suggested that miR-126 and miR-155 might be useful predictive tools in ESRD; however, these results should be validated in a large clinical population, in which comparisons with standard risk factors could be made.

Although the origin of miRNAs present in the circulation is incompletely understood, it is possible that circulating miRNAs are derived from vascular and inflammatory cells in body fluids. For example, endothelial and inflammatory cells can be a source of circulating miR-126 and miR-155. Circulating miRNAs are packed within membrane-bound vesicles (14). These vesicles are byproducts released from cell apoptosis or activation, and are involved in cell-cell communication (28). However, the reduced concentration of circulating miR-126 and miR-155 detected in patients with ESRD was surprising because one would expect that ECs and the inflammatory cell activation that occurs in ESRD contribute to the release of microparticles and remnants of apoptotic cells, thus increasing the expressed levels of circulating miRNAs. However, Neal et al. (26) demonstrated that the rate of miRNA degradation is increased in plasma from patients with severe CKD. Therefore, one may speculate that the reduced circulating miR-126 and miR-155 might be associated with the increased miRNA degradation rate in patients with ESRD.

Additionally, accumulating evidence suggests that apoptotic bodies and microparticles can be transferred to other cell types (29), and that low circulating levels might result from increased delivery of miR-126 and miR-155 to recipient cell, and then act as physiologically functional molecules to exert gene silencing through the same mechanism as cellular miRNAs, suggesting the potentially important roles of circulating miRNAs in the development of CKD. Therefore, it is at least theoretically probable that circulating miR-126 and miR-155 are involved in the development of ESRD.

Moreover, in the present study, we observed that the circulating levels of miR-126 and miR-155 were also significantly reduced in post-HD patients, but did not significantly differ between pre-HD and post-HD, suggesting that the slight changes of plasma miR-155 and miR-126 between pre-HD and post-HD might be associated with the responsiveness to hemodialysis, or heparin anticoagulation may potentially interfere with the RT-qPCR-based assay. Nevertheless, further studies are required for the validation and extension of these results. We also found that circulating miR-155 and miR-126 levels correlated positively with eGFR and hemoglobin but were inversely correlated with phosphate levels. This finding suggested that the reduction of circulating miR-126 and miR-155 might be accompanied by a series of clinical symptoms. Although preliminary, our results showed that the two miRNAs can be considered as 
candidate peripheral markers that may enable the prediction of ESRD progression.

Our study specifically addressed the levels and regulation of inflammation-associated and endothelial-derived miRNAs. Although altered levels of miR-126 and miR-155 in patients with ESRD did not significantly differ between preHD and post-HD patients, miR-126 and miR-155 deserve special consideration as novel biomarkers for risk estimation and classification. Meanwhile, we need to validate these data in large clinical populations. Furthermore, the effect of drug treatment on circulating miR-126 and miR-155 levels

\section{References}

1. Bartel DP. MicroRNAs: genomics, biogenesis, mechanism, and function. Cell 2004; 116: 281-297.

2. Zhang Q, Kandic I, Kutryk MJ. Dysregulation of angiogenesis-related microRNAs in endothelial progenitor cells from patients with coronary artery disease. Biochem Biophys Res Commun 2011; 405: 42-46.

3. Zhu N, Zhang D, Chen S, Liu X, Lin L, Huang X, et al. Endothelial enriched microRNAs regulate angiotensin IIinduced endothelial inflammation and migration. Atherosclerosis 2011; 215: 286-293.

4. Sequeira Lopez ML, Gomez RA. Novel mechanisms for the control of renin synthesis and release. Curr Hypertens Rep 2010; 12: 26-32.

5. Amrouche L, Bonifay R, Anglicheau D. [MicroRNAs in pathophysiology of renal disease: an increasing interest]. Med Sci 2011; 27: 398-404.

6. Shi S, Yu L, Chiu C, Sun Y, Chen J, Khitrov G, et al. Podocyte-selective deletion of dicer induces proteinuria and glomerulosclerosis. J Am Soc Nephrol 2008; 19: 2159-2169.

7. Dai $Y$, Sui W, Lan H, Yan Q, Huang H, Huang Y. Comprehensive analysis of microRNA expression patterns in renal biopsies of lupus nephritis patients. Rheumatol Int 2009; 29: 749-754.

8. Levin A. Clinical epidemiology of cardiovascular disease in chronic kidney disease prior to dialysis. Semin Dial 2003; 16: 101-105.

9. Stinghen AE, Pecoits-Filho R. Vascular damage in kidney disease: beyond hypertension. Int J Hypertens 2011; 2011: 232683.

10. Stenvinkel P, Carrero JJ, Axelsson J, Lindholm B, Heimburger O, Massy Z. Emerging biomarkers for evaluating cardiovascular risk in the chronic kidney disease patient: how do new pieces fit into the uremic puzzle? Clin J Am Soc Nephrol 2008; 3: 505-521.

11. Zampetaki A, Kiechl S, Drozdov I, Willeit P, Mayr U, Prokopi $M$, et al. Plasma microRNA profiling reveals loss of endothelial miR-126 and other microRNAs in type 2 diabetes. Circ Res 2010; 107: 810-817.

12. Mitchell PS, Parkin RK, Kroh EM, Fritz BR, Wyman SK, Pogosova-Agadjanyan EL, et al. Circulating microRNAs as stable blood-based markers for cancer detection. Proc Natl Acad Sci U S A 2008; 105: 10513-10518.

13. Cortez MA, Calin GA. MicroRNA identification in plasma and serum: a new tool to diagnose and monitor diseases. Expert remains to be studied. However, the findings of the current study do allow us to propose that circulating miR-126 and miR-155 levels may be of clinical importance in ESRD.

\section{Acknowledgments}

We thank the patients with ESRD receiving hemodialysis and healthy controls who provided the plasma samples. We thank all the anonymous reviewers for their helpful suggestions for improving our paper.
Opin Biol Ther 2009; 9: 703-711.

14. Arroyo JD, Chevillet JR, Kroh EM, Ruf IK, Pritchard CC, Gibson DF, et al. Argonaute2 complexes carry a population of circulating microRNAs independent of vesicles in human plasma. Proc Natl Acad Sci U S A 2011; 108: 5003-5008.

15. Fichtlscherer S, de Rosa S, Fox H, Schwietz T, Fischer A, Liebetrau $\mathrm{C}$, et al. Circulating microRNAs in patients with coronary artery disease. Circ Res 2010; 107: 677-684.

16. Ai J, Zhang R, Li Y, Pu J, Lu Y, Jiao J, et al. Circulating microRNA-1 as a potential novel biomarker for acute myocardial infarction. Biochem Biophys Res Commun 2010; 391: 73-77.

17. Livak KJ, Schmittgen TD. Analysis of relative gene expression data using real-time quantitative PCR and the 2(-Delta Delta C(T)) Method. Methods 2001; 25: 402-408.

18. Dursun I, Poyrazoglu HM, Gunduz Z, Ulger H, Yykylmaz A, Dusunsel $R$, et al. The relationship between circulating endothelial microparticles and arterial stiffness and atherosclerosis in children with chronic kidney disease. Nephrol Dial Transplant 2009; 24: 2511-2518.

19. Yao $Q$, Lindholm $B$, Stenvinkel $P$. Inflammation as a cause of malnutrition, atherosclerotic cardiovascular disease, and poor outcome in hemodialysis patients. Hemodial Int 2004; 8: 118-129.

20. Fish JE, Santoro MM, Morton SU, Yu S, Yeh RF, Wythe JD, et al. miR-126 regulates angiogenic signaling and vascular integrity. Dev Cell 2008; 15: 272-284.

21. Wang S, Aurora AB, Johnson BA, Qi X, McAnally J, Hill JA, et al. The endothelial-specific microRNA miR-126 governs vascular integrity and angiogenesis. Dev Cell 2008; 15: 261271.

22. Staszel T, Zapala B, Polus A, Sadakierska-Chudy A, KiecWilk B, Stepien E, et al. Role of microRNAs in endothelial cell pathophysiology. Pol Arch Med Wewn 2011; 121: 361366.

23. Martin MM, Buckenberger JA, Jiang J, Malana GE, Nuovo GJ, Chotani M, et al. The human angiotensin II type 1 receptor $+1166 \mathrm{~A} / \mathrm{C}$ polymorphism attenuates microRNA-155 binding. J Biol Chem 2007; 282: 24262-24269.

24. Martin MM, Lee EJ, Buckenberger JA, Schmittgen TD, Elton TS. MicroRNA-155 regulates human angiotensin II type 1 receptor expression in fibroblasts. J Biol Chem 2006; 281: 18277-18284.

25. Zernecke A, Bidzhekov K, Noels H, Shagdarsuren E, Gan 
L, Denecke B, et al. Delivery of microRNA-126 by apoptotic bodies induces CXCL12-dependent vascular protection. Sci Signal 2009; 2: ra81.

26. Neal CS, Michael MZ, Pimlott LK, Yong TY, Li JY, Gleadle JM. Circulating microRNA expression is reduced in chronic kidney disease. Nephrol Dial Transplant 2011; 26: 37943802.

27. Amabile N, Guerin AP, Tedgui A, Boulanger CM, London GM. Predictive value of circulating endothelial microparticles for cardiovascular mortality in end-stage renal failure: a pilot study. Nephrol Dial Transplant 2012; 27: 1873-1880.

28. Tesse A, Martinez MC, Meziani F, Hugel B, Panaro MA, Mitolo $\mathrm{V}$, et al. Origin and biological significance of shedmembrane microparticles. Endocr Metab Immune Disord Drug Targets 2006; 6: 287-294.

29. Prokopi M, Pula G, Mayr U, Devue C, Gallagher J, Xiao $Q$, et al. Proteomic analysis reveals presence of platelet microparticles in endothelial progenitor cell cultures. Blood 2009; 114: 723-732. 